\title{
An Open-Label Tolerability and Actual-Use Human Factors Study of Etrolizumab Autoinjector in Healthy Volunteers
}

\author{
Helen Tyrrell · Renato Ravanello · Jennifer Pulley · Meina Tao Tang • \\ Wenhui Zhang · Mariam Abouhossein · Swati Tole
}

Received: December 23, 2020 / Accepted: February 3, 2021 / Published online: March 29, 2021

(C) The Author(s) 2021

\begin{abstract}
Introduction: Etrolizumab is a novel, dual-action, anti- $\beta 7$ integrin antibody in development for patients with moderate to severe ulcerative colitis or Crohn's disease. Phase 3 studies use a prefilled syringe (PFS) for etrolizumab administration. In parallel, an autoinjector (AI) is being developed to increase delivery options for patients if etrolizumab is approved. Here we describe the overall development strategy and detail the first-in-human study of this AI.

Methods: This open-label study of healthy volunteers evaluated the tolerability and usability of the etrolizumab AI under development. The primary endpoint was the proportion of participants with greater than mild pain following injection. Adverse events (AEs) and usage errors were also assessed. Results were reported by injection site (thigh vs abdomen) and needle
\end{abstract}

Electronic supplementary material The online version of this article (https://doi.org/10.1007/s12325021-01651-8) contains supplementary material, which is available to authorized users.

H. Tyrrell $(\bowtie) \cdot J$. Pulley

Roche Products Limited, Welwyn Garden City, UK

e-mail: helen.tyrrell@roche.com

R. Ravanello $\cdot$ M. T. Tang · W. Zhang ·

M. Abouhossein · S. Tole

Genentech, Inc., South San Francisco, CA, USA training (experienced vs naive). Pharmacokinetic (PK) variability between participants was an exploratory endpoint.

Results: Thirty participants completed the study; $97 \%$ of them did not experience any pain greater than mild, and 50\% did not experience any pain at all. Three usage errors were observed, one of which resulted in delivery of a partial dose of etrolizumab. No patterns of usage errors were observed. Mild injection site reactions (ISRs) were reported; all resolved by the end of the study. Participants injecting into the abdomen reported more ISRs than those injecting into the thigh; needle training did not influence AE incidence or severity.

Conclusions: Results from this first-in-human study demonstrate that single injections of etrolizumab $105 \mathrm{mg}$ using an AI were well tolerated in healthy volunteers, with transient, mild pain and minimal usage errors. Results from this study also informed the design of a subsequent PK comparability study evaluating exposure of etrolizumab administered by either the PFS or the AI. Overall, the availability of an AI may provide an attractive option for patients desiring a convenient, easy-to-use delivery mechanism for etrolizumab.

Trial Registration: NCT02629744

Keywords: Autoinjector; Crohn's disease; Etrolizumab; Inflammatory bowel disease; Ulcerative colitis 
Key Summary Points

Why carry out this study?

Etrolizumab is a novel, subcutaneously administered, dual-action anti- $\beta 7$ integrin antibody in development for patients with inflammatory bowel disease.

An autoinjector (AI) is being developed to increase drug delivery options on approval.

This open-label, first-in-human study assessed the tolerability and usability of an etrolizumab $\mathrm{AI}$ in 30 healthy volunteers.

\section{What was learned from the study?}

$97 \%$ of patients $(n=29)$ never experienced more than mild pain on injection; half did not experience any pain $(n=15)$.

Single injections of etrolizumab $105 \mathrm{mg}$ using an AI were well tolerated in healthy volunteers, and no patterns of usage errors were observed.

\section{DIGITAL FEATURES}

This article is published with digital features, including a summary slide, to facilitate understanding of the article. To view digital features for this article go to https://doi.org/10.6084/ m9.figshare.13688914.

\section{INTRODUCTION}

Inflammatory bowel disease (IBD) is a chronic gastrointestinal disease that severely affects patient quality of life and often results in the need for surgical intervention [1-3]. The predominant forms of IBD are ulcerative colitis (UC) and Crohn's disease, two distinct conditions that share some common symptoms and exhibit a partially overlapping etiology $[4,5]$.
Current pharmacologic therapies for IBD are not curative. In addition, many pharmacologic therapies for IBD lose efficacy over the duration of the disease and can result in systemic side effects $[5,6]$.

Etrolizumab is a novel anti- $\beta 7$ integrin monoclonal antibody in development for patients with UC and Crohn's disease. Etrolizumab selectively inhibits $\alpha 4 \beta 7$ and $\alpha \mathrm{E} \beta 7$ to reduce trafficking of immune cells into the gut and subsequent inflammatory effects on the gut lining [7]. The efficacy and safety of etrolizumab in patients with UC were demonstrated in the phase 2 EUCALYPTUS study [8]. Etrolizumab is now being evaluated in an extensive clinical program of phase 3 studies in patients with moderate to severe UC and Crohn's disease [9] in which etrolizumab is administered once per month by subcutaneous (SC) injection using a prefilled syringe (PFS) with a needle safety device (NSD).

Single-use prefilled autoinjectors (AIs) have many potential advantages over PFS-NSDs, most notably their ability to keep the needle out of sight of the user at all times during injection. AIs also offer increased convenience, ease of use, reduced risk of dosage error, and improved patient comfort. Studies have consistently shown that many patients who self-administer prefer an AI over a syringe-based device [10-13]. For example, a recent study of golimumab in patients with UC demonstrated that most patients preferred administration with an AI than with a PFS, citing increased ease of use and reduced discomfort with injection [13].

The AI currently under development consists of an automated delivery system encasing the same PFS used in the phase 3 studies (Fig. 1). The drug product contained in both the AI and the PFS-NSD consists of a liquid formulation of etrolizumab $105 \mathrm{mg}$ solution $(0.7 \mathrm{~mL}$, nominal volume of $150 \mathrm{mg} / \mathrm{mL}$ ) for single-dose administration. The entire dose is typically administered in about $2 \mathrm{~s}$.

The AI includes many features to improve the patient experience and increase patient comfort with self-administration. The automated drug delivery system is activated by lightly pressing the device perpendicularly onto the skin. Once activated, the AI automatically 
inserts the needle and dispenses the syringe contents. When injection is complete, a needle cover extends and locks over the needle, keeping the needle out of view at all times during injection and protecting the user and others from accidental contact with the used needle. The AI also incorporates visual and auditory mechanisms designed to assist users with selfinjection; a visible spinning top and an audible clicking sound indicate whether drug administration is ongoing or completed. In addition, a visible plunger rod moves across the viewing window while the injection is in progress.

Here we present the overall development strategy for a novel AI for etrolizumab and the findings from a first-in-human study of this device. The primary objectives of this study (NCT02629744) were to evaluate the safety and tolerability of etrolizumab administered by the AI and, primarily, injection site pain after selfinjection and to document critical usage errors. In addition, we evaluated this relatively unique study design, which combined usage error assessments (traditionally conducted as simulated studies) with a tolerability, safety, and exploratory pharmacokinetic (PK) study. A second, two-part, PK comparability study (NCT02996019) was conducted as part of the overall AI development strategy to bridge the exposure data from PFS-NSD to AI for etrolizumab administration. The second study leveraged the exploratory PK results from this tolerability study to refine the study design and final protocol. Results from the second PK comparability study will be reported separately.

\section{METHODS}

\section{Study Design and Procedures}

This first-in-human AI tolerability study was an open-label, single-arm study in healthy volunteers to evaluate pain, safety, and usability of an AI when self-administered subcutaneously. Participants were assigned (1:1) into two groups. To simulate prior experience of self-injection, one group ("needle-experienced") received training before self-injection with the $\mathrm{AI}$; the other group ("needle-naive") did not.
Training involved simulated needle experience by self-injection with placebo, as detailed in "Procedures." Before etrolizumab administration, all participants (irrespective of needle experience group) received an instructions for use (IFU) leaflet regarding the AI for review before self-injection. Participants were randomly assigned to administer study drug to their abdomen or anterior thigh. All participants self-administered a single SC dose of etrolizumab on day 1 of the study in a simulated home setting (Fig. 2). Participants were admitted to the study site on day 1 (where they were monitored during self-injection), remained at the study site until discharge on study day 3, and then returned for follow-up visits on study days $8,29,43,57$, and 85 (study completion).

All procedures performed in studies involving human participants were in accordance with the ethical standards of the institutional and/or national research committee and with the 1964 Helsinki declaration and its later amendments or comparable ethical standards. Informed consent was obtained from all study participants. Study protocol, informed consent forms, information given to participants, volunteer recruitment materials, and all relevant supporting information were approved by the institutional review board (Schulman Associates Institutional Review Board, Cincinnati, $\mathrm{OH}$, USA) before study initiation.

\section{Participants}

Eligible participants were to be between the ages of 18 and 65 years, have a body mass index (BMI) between 18.0 and $32.0 \mathrm{~kg} / \mathrm{m}^{2}$ (inclusive), and be in good health with no significant medical history or laboratory test abnormalities. Both men and women were enrolled, with the target of $55-60 \%$ male participants to mimic the sex distribution of patients with IBD.

Participants with any prior use of anti-integrin therapies (including etrolizumab) or immunosuppressive drugs were excluded, as were participants with a recent history of corticosteroid use and participants with a history of tuberculosis. Participants with prior needle experience were also excluded. 


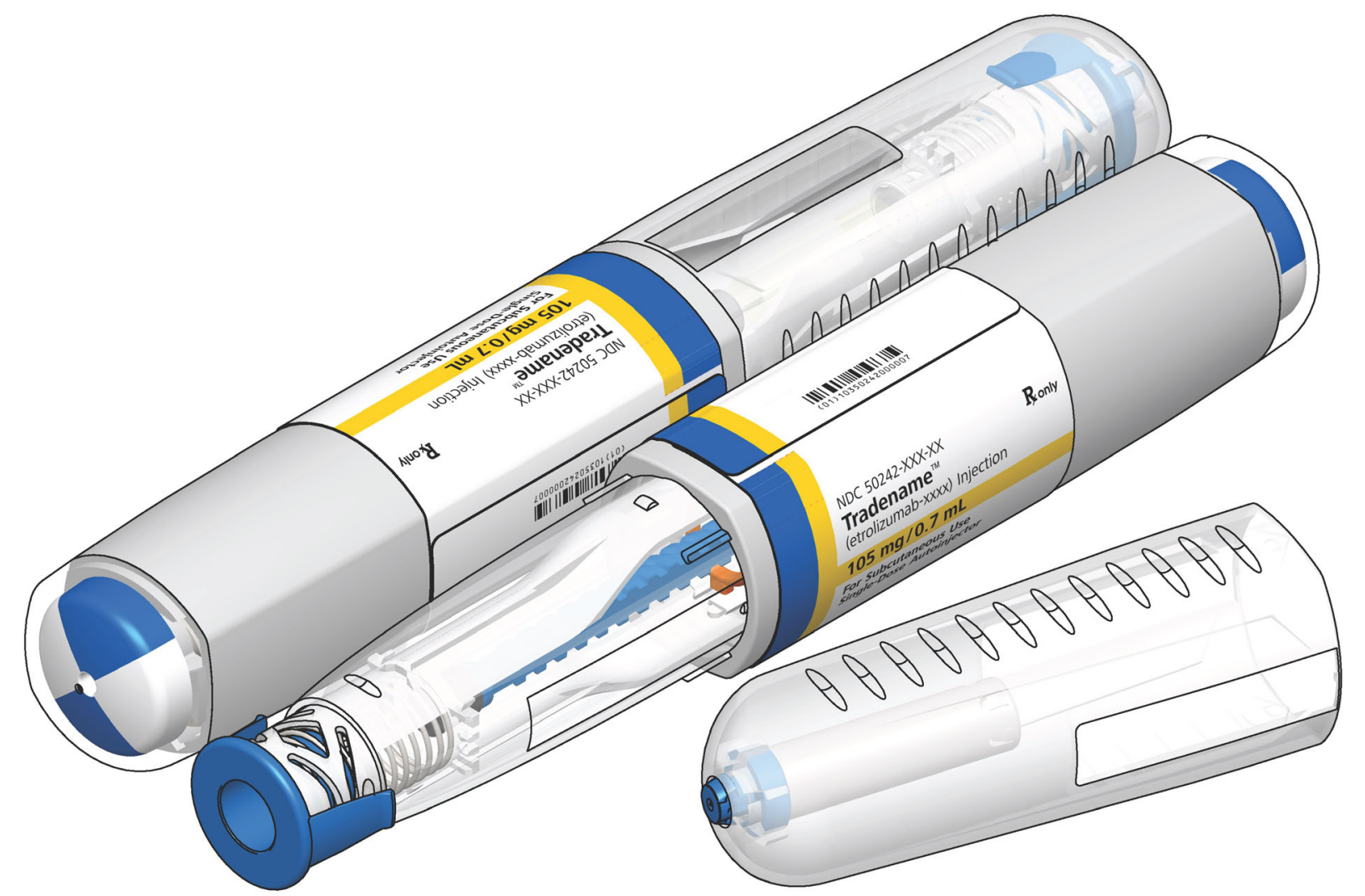

Fig. 1 Prefilled autoinjector of etrolizumab

\section{Procedures}

Participants who received training for self-injection with needle and syringe (simulated selfinjection experience) 5 and 7 days before etrolizumab injection were assigned to the needleexperienced group. During training, participants were instructed by a health care professional on the use of a needle and syringe. Following this instruction, participants practiced self-injection with a placebo solution three times using a needle and syringe. Needleexperienced participants deemed to be suitable by the health care professional (on the basis of their interactions with syringes) progressed through the study.

Needle-experienced and needle-naive participants were randomly assigned, stratified by sex and needle experience, to inject into either the abdomen or the anterior thigh. On study day 1, participants self-administered a single SC dose of etrolizumab $105 \mathrm{mg}$ into their abdomen or anterior thigh using the AI. Participants were assessed for operational difficulties and usage errors and reported pain during and immediately after the injection. One serum sample was taken for exploratory pharmacokinetic assessment 7 days after the SC injection (study day 8).

\section{Approaches for Assessing Pain}

Pain was assessed by two independent methods, both of which were administered by study site personnel. The 7-point categorical Verbal Descriptive Scale (VDS-7) was the primary measure of pain for this study. During VDS-7 administration, participants were asked to choose the number from 1 to 7 that best represented the pain associated with the injection (scale as follows: $1=$ no pain, $2=$ very mild pain, $3=$ mild pain, $4=$ not very severe pain, $5=$ quite severe pain, $6=$ very severe pain, $7=$ almost unbearable pain). As a confirmatory assessment, pain was also assessed on a 


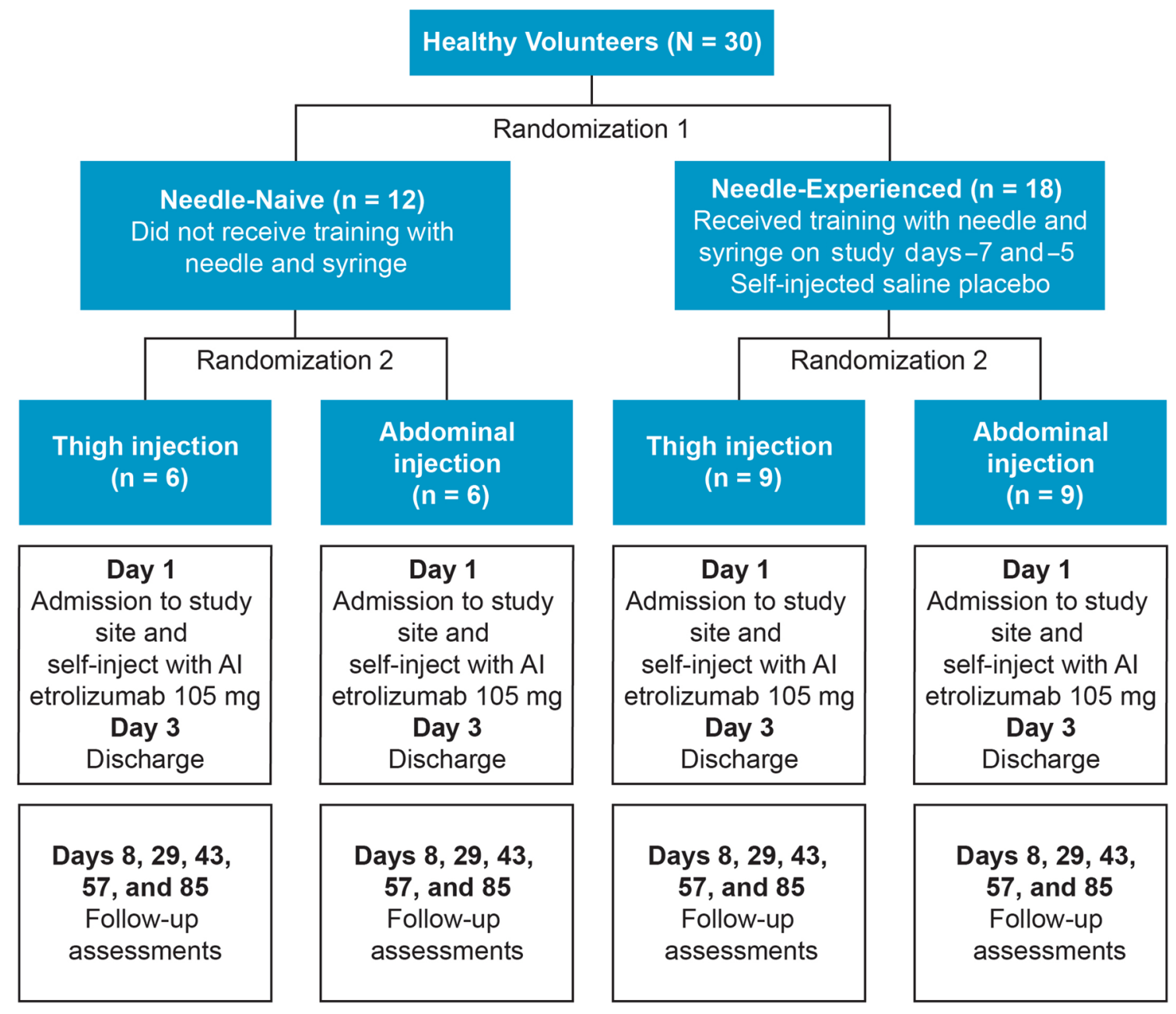

Fig. 2 Autoinjector tolerability and human factors study design. AI autoinjector

100-point continuous visual analog scale (VAS). For the VAS, participants were asked to mark a line on a horizontal $100-\mathrm{mm}$ scale that best represented their pain (scale as follows: $0 \mathrm{~mm}=$ no pain, $100 \mathrm{~mm}=$ worst possible pain). Study personnel then measured the distance between the $0-\mathrm{mm}$ point and the participant's mark to determine their VAS score.

\section{Outcomes}

The primary endpoint was the proportion of participants with greater than mild pain (VDS-7 score $>3$ ) immediately following injection. To meet the primary endpoint, the upper bound of the two-sided 95\% confidence interval (CI) around the proportion of participants experiencing greater than mild pain immediately following injection could not exceed 30\%. Secondary endpoints included the proportion of participants experiencing greater than mild pain at $5,10,20,60$, and $240 \mathrm{~min}(4 \mathrm{~h})$ following injection and the proportion of participants in each VDS-7 category over time.

Tolerability was assessed intensively in this study through active monitoring for injection site reactions (ISRs) on study day 1 at 5, 60, and 240 min after injection and on study days 2,8 , 43 , and 85 . To identify ISRs, a local injection site symptom assessment (LISSA) was performed to assess burning, itching, bruising, redness, formation of hives, and size of the reaction if present. Each ISR was categorized and reported 
as an adverse event ( $\mathrm{AE})$ or a serious $\mathrm{AE}$ as appropriate.

Usage errors and operational difficulties with the AI were documented. In addition, participants' knowledge about the IFU and their overall opinions of the AI experience were collected.

Safety was assessed by AE monitoring, laboratory values, vital signs, physical examinations, electrocardiograms (ECGs), and immunogenicity. For this study, no formal statistical testing was planned.

Determination of PK variability on a single time point of study day 8 following self-injection was assessed as an exploratory endpoint.

\section{RESULTS}

Thirty healthy participants were enrolled and randomly assigned (stratified by sex and needle experience) to inject etrolizumab into the abdomen or anterior thigh. All participants completed the study; however, one volunteer (needle-experienced, thigh injection) did not receive a full dose of etrolizumab because of a usage error. The enrolled population was broadly representative of the IBD population. The median age of enrolled participants was 36 years, the mean BMI was $26.1 \mathrm{~kg} / \mathrm{m}^{2}$, and most participants were white $(n=18 ; 60 \%)$ and not Hispanic or Latino $(n=25 ; 83 \%)$. Approximately half the participants $(n=14 ; 47 \%)$ were male.

\section{Pain and Tolerability}

Half the participants $(n=15)$ did not report pain at any time after injection (Fig. 3). For those who reported pain, all but one reported it as "very mild" or "mild" pain, and most reported that it subsided within $60 \mathrm{~min}$ after drug administration. The primary endpoint was met; only one participant (3.3\%) (95\% CI 0.1-17.2) reported greater than mild pain (VDS-7 score $>3$ ) immediately after the injection that subsided to mild pain at $5 \mathrm{~min}$ after injection. Similar data were reported when using the VAS (data not shown).
Reported pain differed between injection sites. A greater proportion of participants reported pain after injection into the thigh than after injection into the abdomen (60\% vs $40 \%$, respectively) (Fig. 4). The volunteer who reported greater than mild pain was assigned to the thigh administration group. Participants injecting into the thigh also reported longer duration of pain than those injecting into the abdomen; all pain experienced after abdominal injection subsided within $5 \mathrm{~min}$, and most of the pain after thigh injection subsided within $60 \mathrm{~min}$. Needle-experience training did not appear to impact reported pain after etrolizumab injection.

Using the intensive, LISSA-based monitoring scheme described here, 12 of 30 participants (40\%) experienced ISRs during the study; all occurred within $1 \mathrm{~h}$ of etrolizumab injection (Table 1). All reported ISRs were mild (grade 1) and transient, and all resolved by study completion. The most frequent ISR was redness, with a maximum diameter of $31 \mathrm{~mm}$. Most ISRs resolved within $60 \mathrm{~min}$ following injection. One participant reported hives $(18 \mathrm{~mm}$ in diameter) at the abdominal injection site $60 \mathrm{~min}$ after dose; the hives resolved within $3 \mathrm{~h}$ without treatment. Injection site did not appear to affect the frequency or the severity of ISRs.

\section{Usage Errors}

Twenty-seven of the 30 participants (90\%) were able to successfully self-administer etrolizumab using the AI without significant usage errors, regardless of needle experience training. No complaints about the AI were registered, and no pattern of usage errors was observed.

Three usage errors were observed during the study, only one of which occurred during injection. One participant began to remove the AI prematurely during injection, resulting in a droplet of liquid remaining on the participant's skin. Of the two usage errors that did not occur during injection, one participant was unsure when to remove the cap from the AI, and the other incorrectly reported the simulated expiration date. Both usage errors were associated with misunderstanding of the AI labeling and 


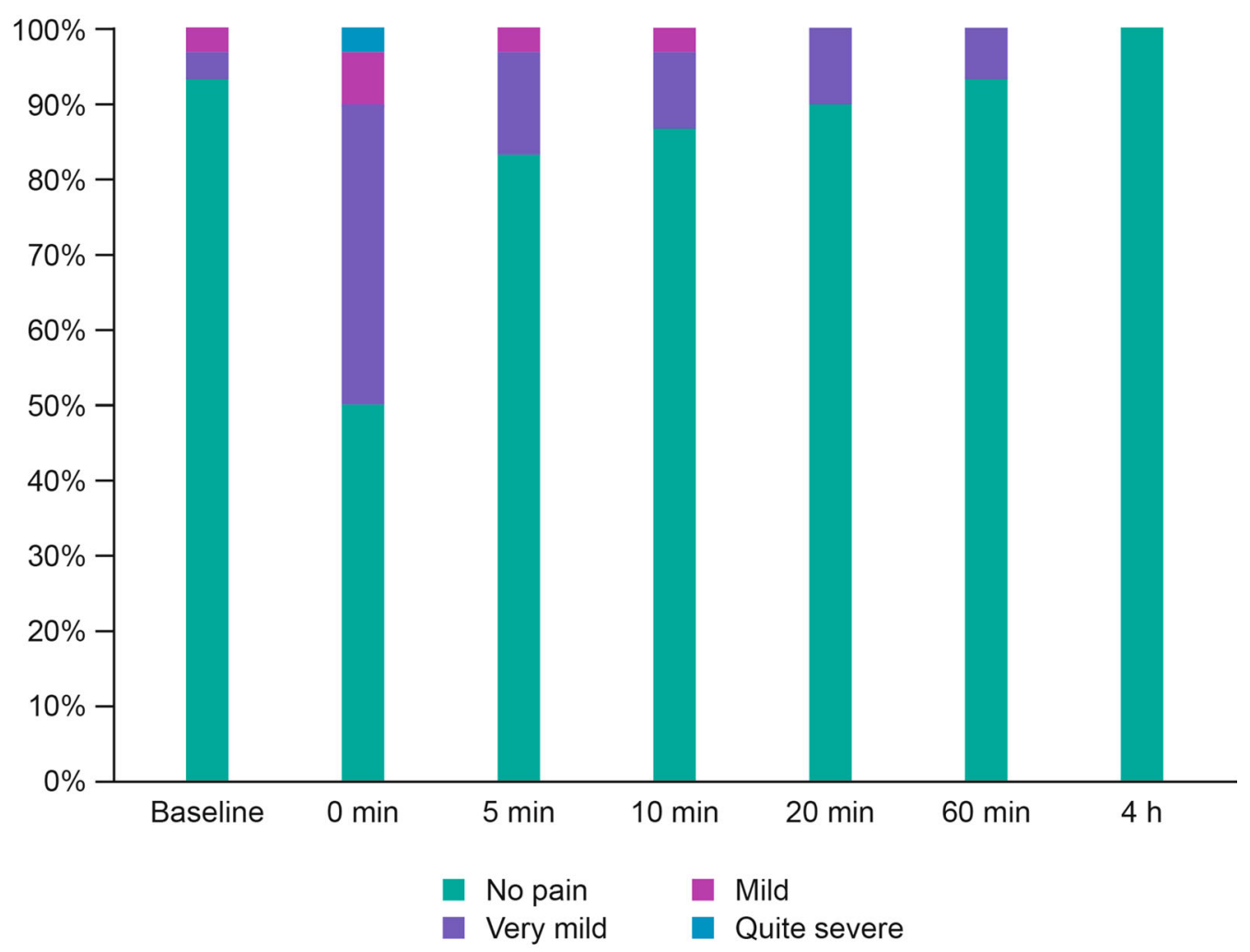

Fig. 3 Pain over time by intensity (7-point Visual Descriptive Scale)

the IFU; neither of these errors impacted the dose of etrolizumab administered.

All participants rated the AI as "very easy" $(n=24 ; 80 \%)$ or "easy" $(n=6 ; 20 \%)$ to use. Most participants reported that the audible and visual feedback mechanisms were "very helpful" $(n=23 ; 77 \%)$ or "helpful" $(n=6 ; 20 \%)$ for determining when the injection had started and stopped and for verifying that a complete dose had been administered. However, some participants $(n=5 ; 17 \%)$ stated that it was difficult to view the visual spinning top during injection into the abdomen. During probes for their understanding of the IFU, some participants $(n=8 ; 27 \%)$ expressed confusion about acceptable injection sites, medication warm-up time, and product storage.

\section{Pharmacokinetics (Exploratory)}

On study day 8 (7 days after injection), the mean ( \pm standard deviation) serum concentration of etrolizumab across all participants was
$13.6( \pm 3.66) \mu \mathrm{g} / \mathrm{mL}$ (median 13.8). Serum concentrations ranged from $5.8 \mu \mathrm{g} / \mathrm{mL}$ to $20.0 \mu \mathrm{g} / \mathrm{mL}$, a roughly $31 \%$ between-participant variability. Neither injection site nor needle training appeared to affect serum etrolizumab concentration at day 8 on the basis of the limited data set.

\section{Safety}

Twenty-nine participants (97\%) received the full 105-mg dose of etrolizumab; one received approximately $90 \%$ of the $105-\mathrm{mg}$ dose. Overall, single 105-mg SC doses of etrolizumab were safe and well tolerated when self-administered using the AI. Sixteen participants (53\%) experienced a treatment-emergent adverse event (TEAE), usually related to the injection site. The most frequently reported TEAEs related to ISRs were injection site erythema $(n=10 ; 33.3 \%$ of participants overall) and injection site pain ( $n=2 ; 6.7 \%$ of participants overall). All TEAEs were mild (grade 1 ) and transient, and all ISRs 

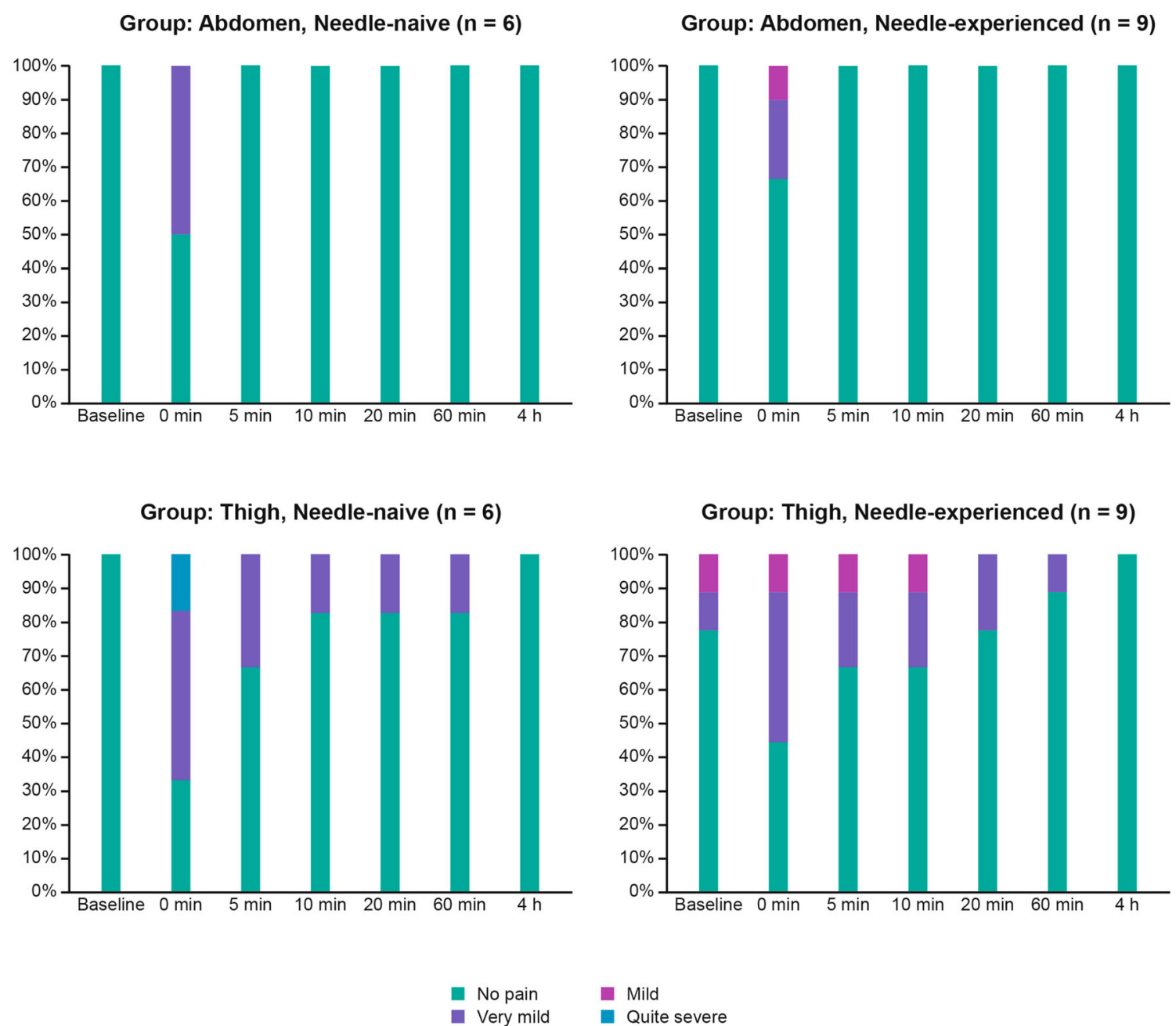

Fig. 4 Pain over time by injection site (7-point Visual Descriptive Scale)

resolved on the first day. No significant changes were noted in clinical laboratory evaluations, vital sign measurements, body weight measurements, or 12-lead ECGs during this study.

Fewer TEAEs were reported by needle-experienced participants than needle-naive participants. A summary of TEAEs can be found in Table S1 in the supplementary material.

\section{DISCUSSION}

In healthy participants, a single self-administered SC dose of etrolizumab using the AI was well tolerated and resulted in no pain or mild pain for most participants. This study met its primary endpoint, with only a single volunteer experiencing greater than mild pain following injection.

Overall, the data presented here are consistent with AI data used in the treatment of other chronic diseases, including rheumatoid arthritis (RA) and chronic kidney disease. These studies suggest that many patients prefer the convenience of an AI to injection with a PFS. Patients commonly report that AIs are associated with less pain than PFSs and perceive AIs as more portable and easier to use [10-12, 14]. A recent study in patients with UC reported similar findings, noting that approximately threequarters of patients in the study preferred injection with an AI than with a PFS [13]. 
Table 1 Summary of local injection site symptom assessments over time by injection site and needle experience

\begin{tabular}{|c|c|c|c|c|c|c|c|c|c|}
\hline \multirow[t]{2}{*}{ ISR } & \multirow{2}{*}{$\begin{array}{l}\text { Time } \\
\text { point, } \\
\text { min }\end{array}$} & \multirow{2}{*}{$\begin{array}{l}\text { Reaction } \\
\text { size, } \mathrm{mm}\end{array}$} & \multicolumn{3}{|c|}{ Abdomen } & \multicolumn{3}{|l|}{ Thigh } & \multirow{2}{*}{$\begin{array}{l}\text { Overall } \\
(n=30)\end{array}$} \\
\hline & & & $\begin{array}{l}\text { Needle- } \\
\text { naive } \\
(n=6)\end{array}$ & $\begin{array}{l}\text { Needle- } \\
\text { experienced } \\
(n=9)\end{array}$ & $\begin{array}{l}\text { Total } \\
(n=15)\end{array}$ & $\begin{array}{l}\text { Needle- } \\
\text { naive } \\
(n=6)\end{array}$ & $\begin{array}{l}\text { Needle- } \\
\text { experienced } \\
(n=9)\end{array}$ & $\begin{array}{l}\text { Total } \\
(n=15)\end{array}$ & \\
\hline $\begin{array}{l}\text { Hive } \\
\text { formation }\end{array}$ & 60 & 18 & & $1(11.1)$ & $1(6.7)$ & & & & $1(3.3)$ \\
\hline \multirow[t]{7}{*}{ Redness } & 5 & 18 & $1(16.7)$ & & $1(6.7)$ & & & & $1(3.3)$ \\
\hline & & 21 & & $1(11.1)$ & $1(6.7)$ & $2(33.3)$ & & $2(13.3)$ & $3(10.0)$ \\
\hline & & 24 & $1(16.7)$ & & $1(6.7)$ & & & & $1(3.3)$ \\
\hline & 60 & $<18$ & & $1(11.1)$ & $1(6.7)$ & & & & $1(3.3)$ \\
\hline & & 18 & & $1(11.1)$ & $1(6.7)$ & & & & $1(3.3)$ \\
\hline & & 24 & & & & $2(23.3)$ & $1(11.1)$ & $3(20.0)$ & $3(10.0)$ \\
\hline & & 31 & & & & $1(16.7)$ & & $1(6.7)$ & $1(3.3)$ \\
\hline
\end{tabular}

$I S R$ injection site reaction

In a recent multinational survey, 200 patients with RA and 100 nurses were asked to rate the relative importance of various components of AIs [15]. Patients and nurses rated "easy to perform the self-injection with the pen (i.e., autoinjector)" as the most important attribute. Other key attributes, as reported by patients and nurses, included "injection needle is safely concealed in the injector body," "audible feedback after completion of the injection," and "visual feedback after completion of the injection"; all these features are built into the etrolizumab AI. Similar results were reported in a European study of 220 patients with RA [16].

The proportion of participants experiencing mild ISRs in this study was higher than that observed in the phase 2 EUCALYPTUS study, in which etrolizumab was administered by study site staff using a vial and syringe [8]. We think this likely reflects differences in the study design because this study assessed tolerability intensively using LISSA to actively monitor for ISRs at scheduled intervals, possibly resulting in overreporting of ISRs.

This first-in-human study is relatively unique in that it combined tolerability assessments, actual-use human factor assessments (such as usage errors), and an initial PK assessment into a single trial. This novel approach aimed, in part, to assess overall risk associated with $\mathrm{AI}$ in a way that would minimize the number of clinical studies necessary, hence reducing the overall time and cost of AI development.

PK assessment was incorporated into the study protocol with a single blood sample taken on study day 8 , around the time of maximum serum concentration following a single SC dose. The intent of this exploratory PK assessment was to understand intersubject exposure variability after etrolizumab SC delivery by AI. In addition, these preliminary PK data helped us evaluate potential differences in exposure following AI injection compared with the predicted exposure using a model generated on the basis of PK data from administration with a vial and syringe in patients with UC.

Of note, the day 8 etrolizumab exposure observed in this analysis was approximately $75 \%$ higher than the predicted value (predicted day 8 median etrolizumab serum concentration $\approx 7.9 \mu \mathrm{g} / \mathrm{mL}$ [90\% CI 4.15-16.3]; data not shown). The PK variability and unexpected higher day 8 exposure from this analysis informed the decision to conduct a two-part study comparing the PK of etrolizumab 
delivered by the AI and by the PFS-NSD in healthy volunteers (Fig. 5).

Results from this study effectively eliminated the requirement for additional AI ease of use studies. In addition, these results influenced the design of a subsequent study to compare PK properties between administration by the PFSNSD and by the AI, mitigating the risk of failing the comparability study and minimizing unnecessary exposure of healthy volunteers to biological treatment.

As a result of the PK findings reported here, a pilot cohort was added to the originally proposed single-part device PK comparability study design. Results from this pilot cohort served to optimize the design of the pivotal cohort by informing the proper sample size, sample collection duration, and body weight range. Information gained from the human factors component of this study resulted in small amendments to the IFU, implemented before the PK comparability study. Results from the device PK comparability study will be reported separately.

This study is not without limitations. This study was designed as an open-label study and included a relatively small number of participants. Although not uncommon for first-inhuman studies, these factors naturally limit the generalization of study results. In addition, as tolerability was a major focus of this study, our study design involved actively probing for tolerability issues, potentially leading to overreporting of AEs. Finally, as this study was focused on the tolerability and human factors of the etrolizumab AI, the study design did not allow for comparisons of the etrolizumab AI with other available AI devices. Additional head-tohead trials comparing the etrolizumab AI with other available devices may be beneficial to further understand its value in clinical practice.

\section{CONCLUSION}

Results from this study demonstrate that single SC injections of etrolizumab with an AI are well tolerated in healthy volunteers and that pain levels following injection are tolerable. Most participants found the AI easy to use, and usage errors were minimal. The AI may be an appropriate delivery mechanism for certain patients with IBD who desire the safety and convenience of self-injection with a concealed needle.

The positive results from this first-in-human tolerability study, in combination with data gathered during the subsequent two-part PK comparability study, constitute a complete development plan to support the use of AI in patients treated with etrolizumab.

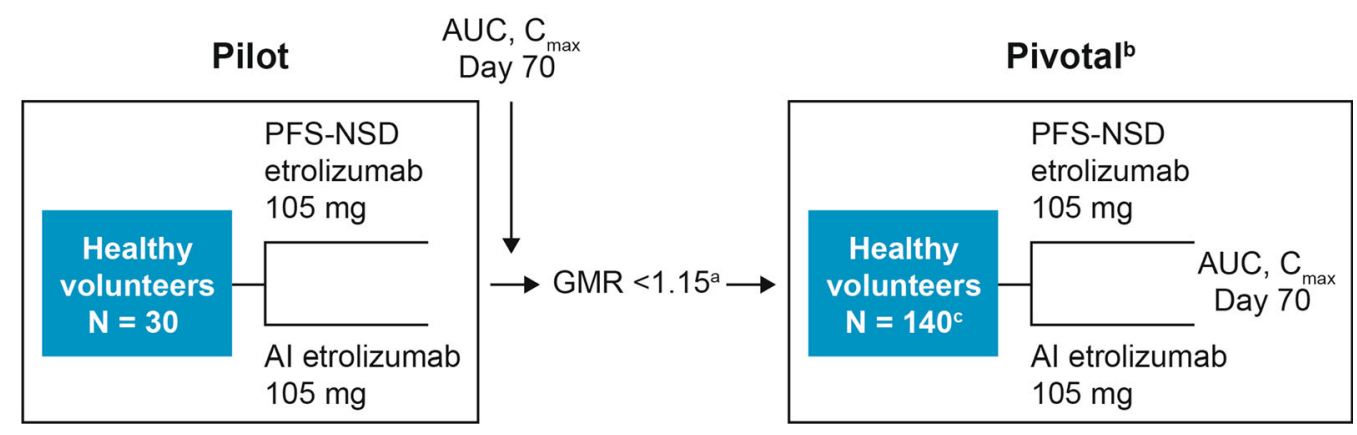

Fig. 5 Two-part pharmacokinetic bridging study design. AI autoinjector, AUC area under the curve, $C_{\max }$ maximum serum concentration of etrolizumab. GMR geometric mean ratio, PFS-NSD prefilled syringe and

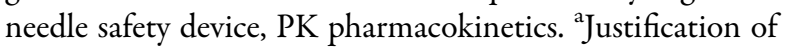
GMR of 1.15 as the decision point was based on the assumption that at $15 \%$ the difference between PFS-NSDs was likely to be real and, therefore, the study would be unable to demonstrate bioequivalence. ${ }^{\mathrm{b}}$ Results from the pivotal study will be reported separately. ${ }^{c}$ Adjustment to $N$ driven by GMR and PK variability from pilot study 


\section{ACKNOWLEDGEMENTS}

The authors thank the participants, investigators, and study site personnel for their valuable contributions.

Funding. Financial support for this study, including the journal's Rapid Service and Open Access fees, was provided by F. Hoffmann-La Roche, Ltd., which funded this study, participated in data analysis and interpretation, and reviewed and approved this manuscript for publication. All authors had full access to all the data in this study and take complete responsibility for the integrity of the data and the accuracy of the data analysis.

Editorial Assistance. Editorial assistance in the preparation of this article was provided by Stacie Dilks, PhD, of ApotheCom (San Diego, CA, USA). Support for this assistance was provided by F. Hoffmann-La Roche, Ltd.

Authorship. All named authors meet the International Committee of Medical Journal Editors (ICMJE) criteria for authorship for this article, take responsibility for the integrity of the work as a whole, and have given their approval for this version to be published.

Disclosures. H. Tyrrell and J. Pulley are employees of Roche Products, Ltd., and receive salary and stock options. R. Ravanello, W. Zhang, M. Abouhossein, and S. Tole are employees of Genentech, Inc., a member of the Roche group, and receive salary and stock options. M. T. Tang was an employee of Genentech, Inc., during the time of the study.

Compliance with Ethics Guidelines. All procedures performed in studies involving human participants were in accordance with the ethical standards of the institutional and/or national research committee and with the 1964 Helsinki declaration and its later amendments or comparable ethical standards. Informed consent was obtained from all study participants. Study protocol, informed consent forms, information given to participants, volunteer recruitment materials, and all relevant supporting information were approved by the institutional review board (Schulman Associates Institutional Review Board, Cincinnati, $\mathrm{OH}$, USA) before study initiation.

Data Availability. Data generated during this study are available from the corresponding author on reasonable request.

Open Access. This article is licensed under a Creative Commons Attribution-NonCommercial 4.0 International License, which permits any non-commercial use, sharing, adaptation, distribution and reproduction in any medium or format, as long as you give appropriate credit to the original author(s) and the source, provide a link to the Creative Commons licence, and indicate if changes were made. The images or other third party material in this article are included in the article's Creative Commons licence, unless indicated otherwise in a credit line to the material. If material is not included in the article's Creative Commons licence and your intended use is not permitted by statutory regulation or exceeds the permitted use, you will need to obtain permission directly from the copyright holder. To view a copy of this licence, visit http://creativecommons.org/licenses/by$\mathrm{nc} / 4.0 /$.

\section{REFERENCES}

1. Casellas F, López-Vivancos J, Vergara M, Malagelada JR. Impact of inflammatory bowel disease on health-related quality of life. Dig Dis. 1999;17: 208-18.

2. Carter MJ, Lobo AJ, Travis SP, IBD Section, British Society of Gastroenterology. Guidelines for the management of inflammatory bowel disease in adults. Gut. 2004;53(suppl 5):1-16.

3. Borren NZ, van der Woude CJ, Ananthakrishnan AN. Fatigue in IBD: epidemiology, pathophysiology and management. Nat Rev Gastroenterol Hepatol. 2019;16:247-59.

4. Zhang YZ, Li YY. Inflammatory bowel disease: pathogenesis. World J Gastroenterol. 2014;20:91-9.

5. Abraham C, Cho JH. Inflammatory bowel disease. N Engl J Med. 2009;361:2066-78. 
6. Rogler G. Gastrointestinal and liver adverse effects of drugs used for treating IBD. Best Pract Res Clin Gastroenterol. 2010;24:157-65.

7. Zundler S, Becker E, Schulze LL, Neurath MF. Immune cell trafficking and retention in inflammatory bowel disease: mechanistic insights and therapeutic advances. Gut. 2019;68:1688-700.

8. Vermeire S, O'Byrne S, Keir M, et al. Etrolizumab as induction therapy for ulcerative colitis: a randomised, controlled, phase 2 trial. Lancet. 2014;384:309-18.

9. Sandborn WJ, Vermeire S, Tyrrell H, et al. Etrolizu$\mathrm{mab}$ for the treatment of ulcerative colitis and Crohn's disease: an overview of the phase 3 clinical program. Adv Ther. 2020;37:3417-31.

10. Kivitz A, Cohen S, Dowd JE, et al. Clinical assessment of pain, tolerability, and preference of an autoinjection pen versus a prefilled syringe for patient self-administration of the fully human, monoclonal antibody adalimumab: the TOUCH trial. Clin Ther. 2006;28:1619-29.

11. Kivitz A, Segurado OG. Humira pen: a novel autoinjection device for subcutaneous injection of the fully human monoclonal antibody adalimumab. Expert Rev Med Devices. 2007;4:109-16.
12. Borras-Blasco J, Gracia-Perez A, Rosique-Robles JD, Castera MD, Abad FJ. Acceptability of switching adalimumab from a prefilled syringe to an autoinjection pen. Expert Opin Biol Ther. 2010;10: 301-7.

13. Vermeire S, D'Heygere F, Nakad A, et al. Preference for a prefilled syringe or an auto-injection device for delivering golimumab in patients with moderate-to-severe ulcerative colitis: a randomized crossover study. Patient Prefer Adherence. 2018;12: 1193-202.

14. Lim WH, Chan D, Boudville N, et al. Patients' perceptions of subcutaneous delivery of darbepoetin alfa by autoinjector prefilled pen versus prefilled syringe: a randomized, crossover study. Clin Ther. 2012;34:1948-53.

15. Tischer B, Mehl A. Patients' and nurses' preferences for autoinjectors for rheumatoid arthritis: results of a European survey. Patient Prefer Adherence. 2018;12:1413-24.

16. Thakur K, Biberger A, Handrich A, Rezk MF. Patient perceptions and preferences of two etanercept autoinjectors for rheumatoid arthritis: findings from a patient survey in Europe. Rheumatol Ther. 2016;3:245-56. 